\title{
Droit et appropriation dans le domaine des biotechnologies. Quelques remarques sur l'évolution récente des pratiques
}

\author{
Maurice Cassier, Jean-Paul Gaudillière
}

\begin{abstract}
Résumé
La nature des brevets accordés dans le domaine des biotechnologies a beaucoup évolué au cours des vingt dernières années avec la multiplication des protections légales concernant des organismes génétiquement modifiés ou la structure de gènes. L'article propose une analyse de ces changements basée sur l'idée selon laquelle les brevets ne constituent qu'une des nombreuses possibilités d'appropriation des connaissances et savoirs-faire scientifiques. Une première partie rappelle la diversité historique des modes d'appropriation qui ont caractérisé le secteur des biotechnologies en analysant trois configurations : le don/contre-don, le monopole instrumental et l'appropriation incorporée dans les matériaux. La seconde partie présente le développement récent de la génétique du cancer du sein pour introduire et discuter trois aspects des nouvelles pratiques : la redéfinition des frontières entre public et privé, la montée des dispositifs formels destinés à gérer les échanges au sein des consortia de recherche et le débat public sur les modalités (et les limites) juridiques de l'appropriation.
\end{abstract}

\begin{abstract}
The nature of patents granted in the biotechnology field has changed a great deal over the past two decades with the proliferation of patents on genetically modified organisms or the structure of genes. This article proposes an analysis of these changes, based on the idea that patents are only one of the many possible forms of appropriation of scientific knowledge and know-how. The first part recalls the historical diversity of the modes of appropriation which have characterized biotechnology, by analysing three configurations: the gift/counter-gift, the instrumental monopoly, and appropriation incorporated in materials. The second part presents recent developments in breast cancer genetics, as a way of introducing and discussing three aspects of new practices: the redefinition of the boundaries between public and private, the increase in formal apparatus for regulating exchange within research consortia, and the public debate on the legal modalities (and limits) of appropriation.
\end{abstract}

\section{Citer ce document / Cite this document :}

Cassier Maurice, Gaudillière Jean-Paul. Droit et appropriation dans le domaine des biotechnologies. Quelques remarques sur l'évolution récente des pratiques. In: Réseaux, volume 16, n88-89, 1998. La propriété intellectuelle. pp. 107-121.

doi : 10.3406/reso.1998.3227

http://www.persee.fr/doc/reso_0751-7971_1998_num_16_88_3227 
BY: $\$$ creative 


\section{DROIT ET APPROPRIATION DANS LE DOMAINE DES BIOTECHNOLOGIES Quelques remarques sur l'évolution récente des pratiques}

Maurice CASSIER, Jean-Paul GAUDILLĖRE 
A u printemps 1988, l'Office américain des brevets décidait d'attribuer à l'Université d'Harvard une protection légale couvrant la production et l'utilisation d'une race de souris rendues susceptibles à la formation d'un cancer par insertion dans les chromosomes de l'animal d'un gène appelé "myc". Trois ans plus tard. en 1991, le biologiste Craig Venter qui travaillait alors pour les Instituts Nationaux de la Santé américains (les NIHs) défrayait la chronique juridique et scientifique en déposant une demande de brevets portant sur de "l'information " génétique, en l'occurrence sur la structure chimique de fragments de gènes humains qui venaient d'être isolés et séquencés mais dont on ne connaissait ni les fonctions ni les usages industriels potentiels (1). Ces deux épisodes sont emblématiques de deux tendances qui ont marqué l'évolution récente du droit des brevets dans le domaine des biotechnologies : d'une part l'appropriation de données génétiques avec la multiplication des brevets portant sur la description de séquences de gènes isolés d'organismes animaux ou humains, d'autre part l'appropriation d'organismes génétiquement modifiés par le biais des technologies de l'ADN recombinant. Si on se souvient que jusqu'à récemment gènes, micro-organismes, plantes et animaux et autres entités biologiques ne pouvaient pas faire l'objet d'une protection par brevet, on mesure l'ampleur des transformations qui ont affecté la définition légale de ce qu'est une invention, un être vivant, ou une découverte utile.

Une explication courante de ces changements consiste à dire que le système des brevets ne fait qu'enregistrer l'évolution des techniques, puisque l'on peut désormais manipuler les gènes comme des molécules chimiques : c'est-à-dire en produisant des formes non naturelles, à la demande et à une échelle industrielle. Cette explication suppose un déterminisme technologique qui n'accorde aucun rôle à l'évolution des pratiques de recherche et aux modalités d'organisation des collectifs savants. De plus, elle ne permet guère de comprendre les enjeux sociaux et éthiques de cette nouvelle jurisprudence. Il faut donc poser autrement la question de ce qui a récemment rendu possible l'appropriation légale "du vivant». Pour cela, nous entendons procéder en deux temps. D'abord, on reviendra sur l'histoire de l'appropriation des produits de la recherche biologique. Ensuite, on partira de l'exemple des gènes prédisposant au cancer du sein pour montrer en quoi le développement actuel de la génomique marque une rupture dans la longue durée des biotechnologies. 


\section{L'appropriation du vivant, un problème de pratiques}

La théorie économique considère généralement la science comme un bien public qui contrairement aux applications technologiques n'est pas susceptible d'appropriation (2). Cette approche de l'innovation fait écho et légitime une vision des brevets telle que ceux-ci représentent des dispositifs économiques et juridiques déconnectés des pratiques de recherche, intervenant a posteriori - une fois que les connaissances ont été établies et validées - pour faciliter (ou non) les transferts de savoirs et leur application dans des sites de production et de mise en valeur qui sont conçus comme essentiellement différents des laboratoires de recherche. Cette approche diffusionniste des rapports entre science et technologie a, depuis une quinzaine d'années, été mise à mal par l'accumulation des études sur l'innovation scientifique, l'imbrication des actions de recherche scientifique et de développement de produits ou de systèmes, ainsi que sur l'histoire des pratiques de recherche (3).

Les nouvelles études sur les sciences permettent en effet de formuler deux thèses fortes:

La science n'est pas par essence un bien " universel », mais un ensemble de produits et d'énoncés instables, définis localement, dont la généralisation et la mise en circulation supposent des investissements matériels, personnels et financiers importants, contrairement à ce que suppose la notion de science comme bien « public ». Les résultats de la recherche sont facilement «appropriables ", parce qu'ils sont en premier licu l'apanage de petits cercles de spécialistes qui disposent des instruments et des savoirfaire qui donnent sens à tel ou tel corpus d'énoncés.

Parce que la science est, dans les premiers stades de son développement, facilement appropriable, les brevets - et plus généralement les dispositifs juridiques définissant droits d'usage et possibilités de réservation ne constituent qu'une partie d'un ensemble plus vaste de pratiques d'appropriation mises en œuvre par les chercheurs à différents moments de leur travail.

L'histoire des sciences biologiques et des biotechnologies permet d'illustrer la prégnance et la diversité de ces pratiques d'appropriation infra- ou para-juridiques.

\section{Première configuration : le don/contre-don}

Dans l'entre-deux-guerres, l'étude de l'hérédité animale a été, aux États-Unis, progressivement dominée par la théorie chromosomique introduite par le groupe de T.H. Morgan (4). Dans ce cadre, les gènes étaient considérés comme des unités élémentaires de contrôle des processus physiologiques dont on ne savait rien, si ce n'est qu'elles étaient alignées le long des chromosomes. Les gènes situés sur les mêmes chromosomes étaient, par hypothèse transmis conjointement d'une génération à la suivante, sauf si intervenaient des « recombinaisons ». Connaître les gènes voulait donc en premier lieu dire que l'on était capable de les localiser sur une carte chromosomique. En pratique, cette localisation voulait dire organiser des milliers de croisements pour déterminer avec quelle fréquence des couples de gènes normalement co-transmis cessaient de l'être. Ce pourcentage étant ensuite considéré comme une mesure de la distance entre gènes. Aux États-Unis, l'épicentre de cette "génétique chromosomique »était constitué par un réseau d'une demi-douzaine de laboratoires travaillant sur un même organisme : la Drosophile ou mouche du vinaigre (5).

La question de la circulation des mutants de Drosophile était un enjeu de première importance pour la vie de ce collectif. En effet, la possibilité d'entrer dans le domaine et le fait de produire un résultat quelconque dépendait fortement de l'accès à ces mutants. La localisation d'un gène était 
fonction des positions relatives adoptées pour les autres gènes, et donc des possibilités de croiser les souches à étudier avec des mouches "porteuses » de mutations déjà connues. En conséquence, à l’inverse de beaucoup de spécialités biologiques où les chercheurs peuvent facilement développer et faire accepter des dispositifs expérimentaux relativement autonomes, la cartographie génétique était (et reste) une activité impliquant une forte intégration des laboratoires.

Dans la mesure où chaque mutant était le plus souvent trouvé, décrit, et reproduit dans un seul site, l'équipe qui avait pour la première fois isolé ou sélectionné une nouvelle souche bénéficiait d'une priorité d'usage et d'un droit - informel, tacite mais réel - d'exclusion des autres. Toutefois, comme l'a montré l'historien R. Kohler, cette capacité potentielle d'appropriation a rarement été actualisée : le contrôle des modalités d'usage d'une souche mutante $n$ 'a qu exceptionnellement donné lieu à des restrictions de la circulation des mouches mutantes. En effet, dans l'économie «morale»du cercle des Drosophilistes, la sanction tacite d'un refus de mise à disposition de mouches intéressant l'un des membres du réseau consistait en une mise à l'écart du cercle des dons. Une règle tacite mais fondamentale de l'organisation du collectif était donc la mise à disposition de la collectivité de tous les stocks de mutants. Cette pratique rendait possible un projet de cartographie qui impliquait à la fois compétition et forte intégration. Il faut signaler que cette forme de "potlach" scientifique allait de pair avec toute une série de pratiques de socialisation et de collectivisation des biens : stages d'apprentissage des techniques, rédaction d'une Drosophila Newsletter informant des changements intervenus dans les laboratoires souscripteurs, etc.

On ajoutera que l'émergence et la stabilité du réseau seraient difficilement compréhensibles sans faire intervenir le contexte sus- ceptible de renforcer la confiance dans la réciprocité de ces échanges : en l'occurrence le fait que les laboratoires du réseau des Drosophilistes étaient pour la plupart dirigés par d'anciens élèves de Morgan ayant en commun un même processus d apprentissage et de socialisation.

\section{Seconde configuration : \\ le monopole de savoir-faire}

Dans l'entre-deux-guerres, l'introduction d'une nouvelle catégorie d'instruments appelés "ultracentrifugeuses » a profondément modifié l'étude d'objets biologiques allant des enzymes aux virus avec cette conséquence que l'ensemble de ces entités a commencé à être considérée comme des "macromolécules ", c'est-àdire des particules formées d'une seule molécule de très grande taille. L'origine des ultracentrifugeuses de laboratoire peut être suivie jusque dans un très petit nombre de laboratoires dont celui de The Svedberg à Upsala, en Suède (6).

Svedberg était un physico-chimiste travaillant sur la nature des transitions entre gel et sol. Au début des années vingt, il songea à mettre à profit le principe de l'écrémeuse à lait - faire tourner un mélange à grande vitesse pour en séparer les constituants - pour analyser les phénomènes d'agrégation dans les solutions de colloïdes. Avec le soutien de la Fondation Rockefeller, Svedberg entreprit de construire une machine imposante par sa taille et son coût et qui comportait non seulement un dispositif de mise en rotation de suspensions à des dizaines de milliers de tours par minute mais aussi un système optique permettant d'observer et de photographier les mélanges en cours de sédimentation. L'usage de cet appareillage amena Svedberg à modifier radicalement sa vision des collö̈des et à propager l'idée selon laquelle les enzymes et protéines biologiques étaient des polymères macromoléculaires dont la masse et la taille pouvaient être calculées à partir des données de centrifugation. 
Jusqu'à la fin des années trente, le centre d'Upsala bénéficia d'un quasi-monopole sur ce type d'instrument. D'une part, parce qu'il s'agissait d'un appareillage dont la construction et la manipulation supposaient des savoir-faire complexes que l'on ne pouvait apprendre que par contact prolongé avec Svedberg et ses ingénieurs. D'autre part, parce que la Fondation Rockefeller adopta une politique restrictive : considérant que la technologie de centrifugation était trop délicate pour une diffusion large, les officiers de la Fondation refusèrent la plupart des demandes de financement de la construction d'appareillage similaire, que ce soit aux USA ou en Europe. La généralisation de la centrifugation et, avec elle, celle du concept de macromolécule protéique passa donc par l'envoi à Upsala des échantillons à étudier et parfois des chercheurs qui s'y intéressaient. Dans ce système, l'appropriation de l'ultracentrifugation par Svedberg reposait moins sur les brevets qu'il avait déposés que sur le contrat moral qui le liait à la Rockefeller en vertu duquel il devait, en contrepartie du soutien de la Fondation, accepter d'étudier les échantillons envoyés par les spécialistes des virus et des protéines. C'est ainsi que les premières « images » de sédimentation du virus de la mosaïque du tabac, le premier virus obtenu à l'état de cristal par Wendell Stanley, un chercheur de l'Institut Rockefeller, furent prises à Upsala. Le monopole d'usage allant avec le monopole d'interprétation, Stanley fut contraint d'accepter l'avis de Svedberg selon lequel les préparations de virus de la mosaïque du tabac étaient composées de plusieurs macromolécules, malgré leur homogénéité de composition chimique.

L'appropriation par monopole d'usage fut progressivement remise en question par l'adaptation à la recherche biologique d'un autre type de centrifugeuse qui avait été construit par deux physiciens américains pour des objectifs très différents. Jesse Beams et Edward Pickles avaient inventé un mécanisme de mise en rotation très rapide de petits dispositifs optiques employés à l'étude de phénomènes physiques de très courte durée. Intervenant à la fois comme des physiciens et des constructeurs d'instru- ments, Beams et Pickles entreprirent de diversifier les usages de leur machine en en multipliant et simplifiant les configurations. Contacté par les spécialistes des virus de l'Institut Rockefeller, Pickles accepta de construire une de ses centrifugeuses puis de venir travailler à l'Institut. À la fin des années trente, l'existence de la machine de Pickles permettait ainsi à Stanley de reprendre les expériences de Svedberg pour en contester les résultats et transformer le virus de la mosaïque du tabac en une macromolécule protéique pure et dotée d'une taille et d'une masse moléculaire unique.

Comme Svedberg, Beams et Pickles avaient déposé des brevets sur leurs ultracentrifugeuses mais sans plus leur accorder de fonction d'appropriation ou de valorisation. Jusqu'à la Seconde Guerre mondiale, les deux chercheurs se contentèrent d'exploiter leur avance technologique. Continuant à développer de nouveaux instruments, ils acceptèrent de donner les plans de leurs modèles, d'aider les autres chercheurs à monter et utiliser leurs machines et parfois même ils entreprirent de construire la totalité d'une machine (moyennant défraiement). Le passage du monopole d'usage au monopole commercial et le recours accru à la protection juridique sont des conséquences de la mobilisation scientifique de guerre. Celle-ci élargit de façon considérable les usages et cristallisa une forte demande d'ultracentrifugeuses utilisées à des fins analytiques mais aussi de préparation de matériaux, par exemple pour isoler des virus vaccins ou des protéines du sang utilisées en transfusion. Peu après la guerre, Pickles quitta l'Institut Rockefeller et créa Spinco, une compagnie spécialisée dans le développement et la construction d'ultracentrifugeuses. Le monopole d'usage de Svedberg était définitivement aboli.

\section{Troisième configuration : l'appropriation incorporée dans les matériaux}

En 1985, l'office américain des brevets étendit à toutes les variétés de plantes le type de protection par brevet qui avait 
quelques années auparavant été accordé aux "inventeurs" de micro-organismes génétiquement modifiés. Cette décision marquait un tournant dans un débat sur les protections à garantir aux sélectionneurs de nouvelles variétés végétales déjà vieux de plusieurs décennies. En 1930, le Congrès américain avait bien adopté un Plant Patent Act (7). Mais celui-ci ne représentait qu'une victoire très partielle pour les "améliorateurs » et producteurs de semences. La loi reconnaissait certes la sélection de végétaux comme une invention et non comme une découverte. Mais, parce que la spécification d'une invention supposait les moyens de sa description et de sa reproduction, le législateur avait choisi de limiter la protection par brevet aux seules variétés horticoles reproduites par multiplication végétative. La loi laissait ainsi hors du champ du brevet la masse des semences agricoles, en particulier les semences de céréales. Pourtant, à l'encontre des attentes des initiateurs de la campagne pour le Plant Patent Act, les semenciers qui auraient dû se sentir floués firent peu pour obtenir la généralisation de la protection. Une des raisons de ce désintérêt tient aux modalités d'appropriation de certaines semences comme les maïs hybrides.

L'invention des mais hybrides est un moment décisif du développement de la génétique agricole aux États-Unis. En 1900), le Département de l'Agriculture adopta un plan de généralisation des tentatives de croisements entre variétés de maiis à mettre en œuvre dans les stations agronomiques bénéficiant de financements fédéraux. Dans ce contexte, un biologiste de la Station d'Évolution Expérimentale fondée par la Carnegie Institution près de New York, H.G. Shull, entreprit de se doter de lignées «pures » par autofécondation forcée de ses maïs. Par là Shull obtint un ensemble de lignées de référence mais il rendit aussi particulièrement visible la " perte de vigueur » que les agronomes attribuaient classiquement à l'autofécondation. Les lignées "purifiées " avaient beaucoup moins de grains et leurs épis étaient beaucoup plus petits que ceux des variétés usuelles. L'hybridation systématique de ces plantes amena ensuite Shull à insister sur le fait que le maïs naturel était en fait composé d'une multitude d'hybrides dont la vigueur était une fonction inverse de la pureté génétique. En effet, à l'inverse des lignées autofécondées, la taille et le rendement en grains des plantes obtenues par hybridation dépassait largement ceux des lignées parentes. Pour rendre compte de cette vigucur des hybrides. Shull et E.M. East, un de ses collègues de la station agronomique du Connecticut, inventèrent la notion d'hétérosis, un effet de synergie dû au simple fait de combiner des patrimoines héréditaires différents.

Parallèlement, Shull proposait de transformer son expérience en un système de production de semences. Dans ce cadre, les stations devraient s'occuper de l'entretien et de l'évaluation des lignées pures qui seraient utilisées pour obtenir des semences hybrides à fort rendement. Le système ne fut jamais mis en xuvre sous cette forme simple, du fait de la difficulté à obtenir des grandes quantités de semences hybrides et de l'importance toute relative des gains de productivité. Néanmoins, une alternative émergea dans les années vingt avec la relocalisation de la production des hybrides des stations agronomiques vers l’industrie et la mise au point par D.F. Jones, un étudiant de East, d'une nouvelle technique impliquant l'intervention pour chaque plant hybride de quatre lignées «pures » et de deux étapes de croisement. Pour des semenciers soucieux de contrôler et de multiplier les usages de leurs produits, les variétés hybrides avaient un immense avantage par rapport aux variétés naturelles : les agriculteurs ne pouvaient pas utiliser les graines issues de la culture des hybrides comme semences car la "vigueur hybride " disparaissait avec la première génération. Autrement dit, les maïs hybrides rendaient la production locale des semences caduque. Comme 
l'écrivaient East et Jones en 1919: avec la méthode des doubles croisements "c'est la première fois dans I'histoire de l'agriculture qu'un sélectionneur va pouvoir obtenir tous les bénéfices d'une de ses inventions ou de quelque chose qu'il a acheté... L'homme qui est à l'origine d'une nouvelle plante qui peut être d'une valeur immense pour l'ensemble du pays n'obtient aucune rétribution - pas même la gloire - car n'importe qui peut la reproduire. En conséquence, il y a peu d'incitation à l'amélioration. L'utilisation des hybrides de première génération permet à l'inventeur de conserver tous les types parentaux et de ne vendre que les graines hybrides qui sont de bien moindre valeur pour la propagation continue. » L'appropriation industrielle était incorporée dans la matière biologique des hybrides.

Une fois l'exclusivité d'usage garantic par la nature technologique des hybrides, la valorisation commerciale supposait encore d'empêcher la reproduction des lignées pures et de convaincre les fermiers d'acheter les nouvelles semences. Le premier résultat fut obtenu par un système policier de surveillance des champs d'expérimentation. Le second par une savante mobilisation scientifique et commerciale qui créa les conditions d'une continuité d'investissements et de sélection, laquelle finit, en retour, par conférer aux lignées hybrides un très fort avantage de productivité.

Après avoir rappelé l'ancienneté et la diversité des modes d'appropriation dans le champ des biotechnologies, venons-en aux transformations associées à la généralisation des pratiques de clonage, séquençage et recombinaison de gènes.

\section{Les pratiques d'appropriation de la science dans le champ de la génomique}

Les années 1990 ont été marquées par la diffusion des pratiques d'appropriation dans le domaine de la recherche génomique dans un contexte de resserrement des liens entre la science, la médecine et le marché. Plus d'un millier de brevets sur des fragments de gènes ou sur des applications diagnostiques et thérapeutiques dérivées de la connaissance des séquences de gènes ont été déposés à ce jour. Les contrats de recherche entre les laboratoires pharmaceutiques et les laboratoires publics, assortis de clauses de confidentialité et d'exclusivité, se sont multipliés. Des intérêts privés interviennent directement dans le décryptage du génome et dans la recherche de gènes de prédisposition aux maladies. souvent pour le compte des grandes firmes pharmaceutiques. Les échanges de bio-matériels et de données non publiées entre les laboratoires ont été soumis à des restrictions (8).

Le domaine du cancer du sein offre un terrain de choix pour étudier la diffusion des pratiques d'appropriation, d'une part parce que ces travaux se situent à la charnière de deux domaines daction scientifique et technique - la génétique et la clinique - et d'autre part parce que les résultats des travaux entrepris sur les gènes contrôlant la prédisposition à telle ou telle forme de cancer sont susceptibles d'avoir des applications commerciales étendues (9). La recherche des gènes de prédisposition aux maladies suppose tout d"abord de disposer de collections de matériel génétique et de données médicales sur des familles de patients. La possession ou l'accès à de telles collections joue donc un rôle critique dans le développement des recherches sur les gènes. C'est un moyen d'obtenir ou de préserver une avance sur les autres compétiteurs. Au milieu des années 1980, des chercheurs français, notamment Gilbert Lenoir du Centre International de Recherche sur le Cancer et des Hospices civils de Lyon. ont eu accès à un gisement de données génétiques et cliniques de grande qualité auprès d'un clinicien américain de I'Utah. H.T. Lynch. qui avait com- 
mencé à engranger des données sur le cancer dès les années 1960. Au premier abord. les conditions de mise en circulation de ces matériaux rappellent le potlach des Drosophilistes. De nombreux chercheurs ont eu la possibilité de consulter librement la collection de fiches familiales patiemment établies par Lynch et son assistante (plus de 100() familles répertoriées) et de faire des prélèvements d'ADN éventuellement ramenés en France.

Cependant, les modes d'organisation de la recherche et donc les conditions d'intégration sont assez différentes de celles qui dominaient les premiers réseaux de cartographie chromosomique. Les mêmes chercheurs français ont dès l'origine participé à un consortium international sur le cancer du sein qui se réunissait tous les ans pour échanger des données. Le premier gène de prédisposition au cancer du sein, BRCA1, a été repéré par M.C. King de l'Université de Californie à Berkeley, en 1990, grâce à la collaboration de familles comptant plusieurs cas de cancer du sein. C'est un autre chercheur américain, Mark Skolnik, de l'Université de l'Utah, qui parvint à séquencer le gène, en 1994. Skolnik s'était très tôt engagé dans la création d'une start up, Myriad Genetics, soutenue par le laboratoire pharmaceutique Elli Lily. Après le séquençage, Myriad Genetics a déposé plusieurs brevets sur BRCAl. Mais la compagnie se trouve concurrencée par une autre société de biotechnologie américaine, OncorMed. Avec l'intensification de la course au gène, la participation des chercheurs américains au consortium de recherche international s'était faite moins régulière. La recherche d'un second gène de susceptibilité qui expliquerait les formes familiales de cancer du sein où BRCAl ne joue pas de rôle apparent se fit alors sans référence à une coordination mondiale. Parallèlement aux initiatives américaines, un consortium européen, soutenu par l'UE, se mit en place pour réunir une collection de données génétiques de grande taille (214 familles) et centraliser les études statistiques. Son fonctionnement conjugua coopération et compétition. Si les partenaires s'accor- dèrent pour envoyer leurs données puis leurs échantillons à l'Institut de Recherche sur le Cancer de Sutton en Grande-Bretagne qui disposait d'une forte compétence en bio-informatique et de plus grandes capacités de séquençage, ils essayèrent, chacun dans leur coin, d'identifier le gène. Pendant la phase la plus aiguë de course au gène BRCA2, les échanges horizontaux entre les laboratoires européens furent suspendus. Seuls les échanges verticaux entre ces laboratoires et I'Institut de Sutton perdurèrent. Les liens horizontaux ne reprirent qu'une fois le gène $\mathrm{n}^{\circ} 2$ séquencé et protégé. Une asymétrie se développa au sein du consortium, puisque le centre de recherche de Sutton, qui recueillait les données de tous les participants pour en faire l'analyse statistique, disposait d'un monopole d'expertise et de l'ensemble de la collection des données. C'est lui qui déposa la demande de brevet sur BRCA2. L'appropriation était fondée ici sur le contrôle d'un outil de recherche et la centralisation consécutive des données.

La bataille des brevets est en cours (on ne compte pas moins de 10 demandes déposées sur BRCAl et BRCA2; 3 brevets ont été accordés à ce jour, à OncorMed et Myriad Genetics sur BRCA1 et aux Anglais de Sutton sur BRCA2). Un premier procès oppose OncorMed et Myriad Genetics. Quant à la propriété industrielle de $\mathrm{BRCA} 2$, elle est revendiquée à la fois par les Européens, en l'occurrence le Cancer Research Campaign Technology, et par les Américains de Myriad Genetics (qui auraient bénéficié d'une fuite en provenance du consortium européen). Bien avant l'obtention de son premier brevet, en juillet dernier, Myriad Genetics avait développé des tests de repérage des mutations les plus fréquentes de BRCAl que la compagnie offre directement aux patients dans son propre centre de soins pour un prix de 600 dollars. On voit ici clairement que la stratégie d'appropriation de Myriad Genetics mobilise le droit des brevets, mais ne s'y limite pas. L'appropriation de la rente d'innovation associéc à la connaissance de la séquence de BRCAl repose sur l'avance dans la mise au point et la mise en 
œuvre de tests diagnostiques. cest-à-dire sur l'information accumulée à propos des mutations de gènes rencontrées dans une population de patients de plus en plus étendue et sur la maîtrise de procédures de tests assez complexes. Pour autant, comme dans le cas du monopole d'usage de l'ultracentrifugeuse, Myriad Genetics pourrait voir sa prépondérance et donc sa part de rente fondre comme neige au soleil si le kit d'analyse des mutations proposé par une nouvelle société américaine, Affimetrix, se révélait fiable. En effet, ce nouveau kit, d'utilisation beaucoup plus simple, pourrait être diffusé directement auprès des praticiens des consul-tations de cancérologie.

Cette tentative d'appropriation (et de valorisation) par contrôle des techniques de "soins " dérivées de la génomique amène à souligner un autre enjeu qui est celui du rapport aux professionnels de santé. Les sociétés privées comme Myriad Genetics ne se contentent plus de fournir des kits aux services hospitaliers. Dans la mesure où elles maîtrisent des techniques de diagnostic relativement complexes, elles sont tentées de court-circuiter les services cliniques et d'étendre leur emprise sur l'offre de soins. Cette stratégie, qui tente de réduire le rôle des professionnels de la santé, n'est pas sans poser de problèmes : en amont, il faut sélectionner les patients pour les tests, et en aval, il faut les conseiller et les aider pour recevoir les résultats. La situation est très différente en France, où ce sont les institutions universitaro-hospitalières qui ont développé nombre de techniques de repérage des mutations génétiques, en association étroite avec l'ouverture de consultations de génétique du cancer. La mise au point de ces tests étant couverte par les fonds prévus pour la recherche dans le budget global de l'hôpital, ces laboratoires cliniques se sont regroupés en consortium pour échanger et normaliser leurs techniques. Il existe un certain recouvrement entre ce consortium de standardisation des techniques de mutation des gènes et le consortium européen qui a fait la chasse à BRCA2. Il reste à savoir comment seront réglés les problèmes de propriété et de royalties entre les détenteurs de titres de propriété industrielle sur les séquences et ces groupes hospitaliers qui mettent au point des tests génétiques dans leurs propres réseaux de recherche et d'intervention clinique. Les laboratoires hospitaliers sont aussi susceptibles de se lier avec des laboratoires pharmaceutiques qui souhaitent accéder à leurs collections de matériel génétique pour tester leurs techniques en développement. Les contrats peuvent prévoir des contreparties sous forme de mise à disposition de matériel de recherche.

Pour compléter ce tableau des pratiques d'appropriation dans le champ du cancer du sein, il faut mentionner l'intervention des associations de patients contre l'attribution de brevets sur les gènes de susceptibilité, aux États-Unis et en Grande-Bretagne. L'association Breast Cancer in Britain a notamment déclaré : "Il est anormal que les femmes aient à payer de fortes sommes pour ce test. Et il est scandaleux que quelqu'un puisse réclamer la propriété sur quelque chose qui appartient à quelqu un d'autre. »

L'exemple des gènes de prédisposition au cancer du sein permet donc de repérer :

1 - l'émergence de nouvelles formes d'organisation de la recherche qui associent étroitement, dans des accords bilatéraux ou des consortia de recherche ou de standardisation des techniques, des laboratoires publics, des sociétés privées de biotechnologie, des laboratoires pharmaceutiques, ainsi que nombreuses institutions hybrides. scientifiques et médicales, comme I'Institut Curie ou le Cancer Research Campaign Technology,

2 - la montée des pratiques d'appropriation parmi les acteurs scientifiques (sur les 10 demandes de brevets sur les gènes de prédisposition au cancer du sein, 5 émanent d'universités et les 4 brevets déposés par Myriad Genetics impliquent des institutions universitaires ou médicales).

3 - la forte mobilisation du droit des brevets dans les stratégies d'appropriation, même si les acteurs utilisent, en amont ou en aval des brevets, le contrôle de laccès aux collections de données familiales et 
d`ADN, la maîtrise des outils de recherche ou le contrôle des usages des techniques.

4 - les controverses ouvertes dans l'espace public sur la brevetabilité des gènes, sur plusieurs registres, scientifique et industriel (contester l'étendue des revendications des brevets sur les gènes qui freineront le développement des recherches). sanitaire et civique (contester lattribution d'un monopole sur une invention qui devrait être très largement accessible aux patients), économique et moral (mettre en cause le brevetage d'entités considérées comme appartenant au patrimoine commun de l'humanité).

Arrêtons-nous ici sur trois problèmes théoriques relatifs à ces nouvelles formes d'appropriation de la science et des techniques.

\section{La redéfinition de la frontière entre le domaine public et le domaine privé de la recherche}

Nous avons vu à propos du cancer du sein l'intérêt croissant des capitaux privés pour la recherche sur le génome (10). Dans le même temps, les institutions de recherche publique jouent un rôle essentiel dans le brevetage des produits des recherches sur ce génome (11). Certains auteurs pensent que la rencontre entre la science et l'économie, particulièrement dans le domaine du génome. se réduit à un mouvement unilatéral de privatisation (12). Nous pensons que les évolutions en cours sont plus ambivalentes et laissent place à une pluralité de compromis entre le public et le privé. Tout d'abord, les accords conclus entre les laboratoires et les firmes ménagent des compromis variés : le contrat entre le TIGR et la société Human Genome attribue à l'industriel des droits exclusifs sur les résultats. tandis que les universitaires sont autorisés à publier. éventuellement de manière différée si l'industriel le demande : le contrat entre l'Institut de Génétique et de Biologie moléculaire de Strasbourg et la firme BMS est plus ouvert, puisqu'il n’accorde à l'industriel qu'un droit aux brevets sur certains projets et un droit de première lecture des résultats avant publication.

Ensuite, les institutions scientifiques ne sont en aucune manière entièrement absorbées par la sphère privative. Elles ont en fait appris à gérer la protection de certains résultats d`intérêt industriel, le partage des données avec leurs partenaires proches et la publication dans les réseaux académiques. Mieux, les sociétés privées de génomique publient également dans les revues scientifiques, moyennant un certain délai, ne serait-ce que pour valoriser leurs titres sur les marchés financiers et pour attirer des partenaires académiques réputés. Certaines initiatives, tant publiques que privées, visent à rétablir un meilleur équilibre entre le domaine public et le domaine privé de la recherche. En effet, une privatisation excessive des données conduit à une redondance croissante des collections d'informations et à une ignorance mutuelle des données possédées par les uns et par les autres. Cela se traduira à terme par de coûteuses disputes sur la priorité des inventions. Aussi le laboratoire de Los Alamos a-t-il souhaité créer une banque de données qui permet aux producteurs de soumettre leurs nouveaux résultats de façon confidentielle, afin qu'on puisse leur dire si quelqu'un travaille sur les mêmes sujets. La firme pharmaceutique privée Merck a décidé de verser dans le domaine public les séquences obtenues dans le cadre de diverses collaborations avec les laboratoires universitaires, ce qui lui permet de s'opposer aux tentatives d'appropriation qui portent sur les connaissances amont et de bénéficier d’éventuels retours en laissant les données dites de base

(10) ('e point è Également souligné par l'OCDE: 1995

(11) CORONINI et al. 1997. montrent que parmi les 1.5 premiers déposants de brevets sur le genome de par le monde, 7 sont des institutions de recherche et de santé (I)épartement américain de la santé. Liniversité de Californie. Hopital Général de Boston, Institut Pasteur, Cniversité du Texas. Inserm. Max-Planck Institute).

(12) HERMITTE, 1993: DAS(jLPTA DAVII), 1994. 
librement accessibles à la communauté scientifique. On observe actuellement une course de vitesse entre brevet et publication des séquences (13). Soulignons enfin l'émergence de données collectives (14), caractéristiques des nouvelles formes d'organisation de la recherche en consortia ou en réseaux coordonnés. Ces données ont un statut hybride, à mi-chemin du public et du privé. Elles sont partagées entre les partenaires afin de stimuler l'invention collective. Elles ne sont pas accessibles aux chercheurs extérieurs au réseau. Elles concernent notamment des données de recherche intermédiaires qui sont utilisées comme inputs de futures recherches. À la différence des échanges entre scientifiques qui sont régulés par des normes tacites de réciprocité, les participants, souvent hétérogènes, s'entendent sur des jeux de règles explicites.

\section{La montée des pratiques d'appropriation formalisées ou juridicisées}

Tandis que les pratiques d'appropriation étudiées plus haut faisaient une plus large place aux accords tacites, aux monopoles de fait fondés sur le savoir-faire et à la localité des techniques, les acteurs de la génomique mobilisent largement le droit du brevet et les contrats. Pour expliquer ce phénomène, on peut invoquer l'intensité de la compétition économique sur le sujet et la nécessité de délimiter les droits des nombreux acteurs hétérogènes qui participent au développement des innovations dans ce secteur (les institutions scientifiques et médicales, les sociétés privées de génomiques, les laboratoires pharmaceutiques). Pour les institutions scientifiques et les sociétés de génomiques, le dépôt de brevets est le moyen de valoriser leurs actifs sur le marché et de nouer des coopérations avec les laboratoires pharmaceutiques. Il nous semble toutefois qu'il faut aussi faire intervenir dans l'explication les pratiques des généticiens : il est difficile aujourd hui pour un acteur de maintenir un contrôle fort sur les données génétiques autrement qu'en recourant à des obligations contractuelles ou juridiques. Tout d'abord, ces données sont pour une large part codifiées et inscrites sur des supports papier et informatique. Leur accessibilité physique est de ce fait très élevée (15). Tous les utilisateurs potentiels peuvent y accéder instantanément sur les réseaux de données, une fois qu'elles y ont été déposées. Ensuite, dans la mesure où elles circulent dans un réseau scientifique et industriel qui est désormais nombreux et multiforme mais aussi bien équipé et à forte densité d'expertise, le coût de reproduction et d'utilisation des séquences d'ADN est relativement bas. Il est donc plus difficile que dans d'autres domaines d'exercer un monopole de fait, fondé sur le savoir-faire ou le contrôle de l'outil de recherche. Il devient indispensable de recourir au droit.

Paradoxalement, cette large dissémination des sources et des données de recherche est l'une des causes de la fragilité des brevets déposés sur le génome. Dans la mesure où, sur un sujet donné, les compétiteurs sont très proches les uns des autres, les conflits sur l'antériorité des inventions sont nombreux. Et, pressés par la concurrence et par la course aux publications, les acteurs sont tentés de «prendre acte " en déposant des brevets sur des portions de génome mal définies et sur des applications insuffisamment décrites (au sens où elles sont rapidement contestées). La solidité des brevets déposés sur le génome est d'ailleurs une source d'interrogations explicites des acteurs impliqués dans le domaine qui tracent les limites de l'analogie fréquente

(13) OCDE, 1995.

(14) CASSIER, 1998.

(15) Il est désormais possible, pour les laboratoires ou des cabinets de conseil en propriété industrielle, de se brancher sur les bases de données publiques pour faire des manipulations sur informatique, sans passer, ou avant de passer, aux manipulations physiques. Les expériences mobilisent alors de l'ADN informatique, des sondes el des enzymes électroniques. 
entre génomique et génie chimique. En premier lieu. les cadres d'interprétation du droit du brevet appliqué à cette nouvelle classe d'objets (des fragments de gènes et des techniques médicales dérivées) sont encore fragiles. On s"interroge encore sur la brevetabilité des séquences partielles de gènes, sur la définition de l'activité inventive, sur l'applicabilité industrielle des inventions revendiquées. En second lieu, l'objet breveté est lui-même sujet à incertitude : dans le domaine des gènes de prédisposition à une affection, le brevet est basé sur la revendication d'un lien statistique entre une portion de génome, plus ou moins large et bien définie, et une maladie. Le risque est grand que ce lien statistique se distende ou soit remis en cause au fur et à mesure que de nouvelles populations sont analysées et que les recherches avancent. Un concurrent peut proposer une région plus étroite. le gène lui-même, ou redéfinir le lien statistique. Qui plus est, on peut s'apercevoir que le gène revendiqué n'occupe qu'une place mineure dans une cascade de gènes, etc. En troisième lieu, il existe une incertitude sur la définition de l'équivalence, et donc de la nouveauté, des objets revendiqués : comment déterminer qu'une protéine est nouvelle par rapport à une protéine déjà décrite ? Faut-il fixer un nombre d'aminoacides que l'on a modifiés? Ou prendre en compte, au cas par cas. la modification des propriétés et des effets de la molécule? En quatrième lieu. ces brevets sont menacés par l'insuffisance de description des inventions, notamment pour les revendications qui portent sur des thérapies géniques. Dans la mesure où on n'a pas les moyens de décrire suffisamment ce type dapplication industrielle, le risque existe de rédiger des "brevets de papier ", sans dispositif technique probant. En cinquième lieu. les brevets sont contestés par des associations de patients ou des mouvements écologistes (16), qui se situent sur plusicurs registres de justification : ils interviennent sur les critères classiques de brevetabilité (l'étendue des revendications, la définition de l'activité inventive), mais aussi sur le droit de monopole commercial conféré par le brevet sur des entités a priori inappropriables comme les gènes.

\section{Le droit des brevets dans l'économie et la société}

Les controverses sur l'extension du champ de la brevetabilité au génome, et plus largement au vivant et à l'humain, font apparaître un conflit entre deux logiques. D'un côté, certains conçoivent le droit des brevets comme un droit technique, pratiqué par des spécialistes, les ingénieurs-brevets, qui sont proches des sollicitations du marché. Alain Gallochat, responsable de la propriété industrielle à l'Institut Pasteur, revendique une nette séparation entre le droit des brevets, d'une part, et les problèmes éthiques et les instances politiques, d'autre part. Les spécialistes du droit de la propriété industrielle ont pour mission de se préoccuper de la définition et de l'application des critères de brevetabilité classique, tandis que les instances publiques ou les associations régulent ensuite les usages sociaux des inventions brevetées (par exemple, l'Agence pour le Médicament). D'un autre côté, des juristes entendent réinscrire la question de la brevetabilité des produits dérivés du génome dans les débats sociaux et éthiques sur les usages des techniques et sur le statut du corps humain, des animaux, etc. (17). Auquel cas, les spécialistes de la propriété industrielle ne doivent pas constituer une sphère autonome, à l'écart des débats sur les choix sociaux. Les premiers, à l'instar d'Alain Gallochat, militent pour une extension rapide du droit des brevets aux inventions des biotechnologies. Ils s inscrivent dans le court terme du marché et de la compétition entre 
l'Europe et les États-Unis. Les seconds, à l'instar de Marie-Angèle Hermitte. privilégient le long terme et se fondent sur un principe de prudence.

Loin de porter seulement sur les limites de la propriété intellectuelle et les conditions de rétribution des inventeurs, ces controverses touchent à la définition des rapports entre économie et société et plus précisément à la définition de nouvelles modalités de régulation des usages des produits de la génomique qui ne se limitent pas aux normes de bonne pratique des praticiens (médicaux ou industriels). On notera en guise de conclusion que des juristes ont - de ce point de vue - avancé des compléments ou des alternatives au droit actuel des brevets. J.-C. Gallou, par exemple, propose un régime de droits superposés : si le génome humain est un bien commun à l'humanité, il serait possible de détenir un droit privatif sur une application associée à un gène : "Ainsi, la qualification de patrimoine commun de l'humanité est-elle une qualification juridique qui coiffe toutes les autres, mais qui les laisse toute subsister (18). »C. Labrusse-Riou propose la création de nouvelles catégories juridiques comme celle de "bien commun ». Le bien commun se distingue à la fois du bien privé et du bien public : " L'infinie variété de régimes particuliers à certains biens, privés ou publics, montre qu'il y a place pour de nouvelles catégories intermédiaires entre les biens publics et les biens privés : la vie. le corps pourraient être érigés en "biens communs" (19). " Les biens communs échappent à l'appropriation patrimoniale et commerciale, mais ils peuvent faire l'objet d'une gestion finalisée pour une destination thérapeutique, soit un intérêt collectif bien délimité et contrôlé (une thérapie stricto sensu à l'exclusion des cosmétiques, par exemple). L'appropriation ne peut être que finalisée, pour certains usages, et provisoire. La rente d'innovation prélevée ne doit inclure que le coût des investissements, à l'exclusion de la matière première, gratuite. Elle doit être en partie affectée à "des ceuvres d'intérêt général ». Un tel régime juridique s'efforce de concilier des incitations fondées sur une appropriation limitée et la maîtrise sociale des usages des inventions. 
ALLEN G. (1975), The Life Sciences in the Twenticth Century, Cambridge : Cambridge University Press.

BUGOS G.E. and KEVLES D. (1992). « Plant as Intellectual Property : American Practice, Law and Policy in a World Context », Osiris. 7, 75-104.

CALLON M. (1994), «Is Science a Public Good ? ". Science, Technologie and Human Values, 19 (4), 39.5-424.

- (1995), «Externalités et politiques publiques : le point de vue d'un sociologue ", Colloque CNRS, Innorations technologiques et performances : approches interdisciplinaires, Paris, 71-84.

CASSIER (M.) (1998), « Lémergence de nouvelles formes d'invention collective : réscaux et consortia de recherches dans le domaine des biotechnologies », à paraître dans Annales des Mines. 1-10.

COHENDET P., HÉRAUD J.A. et ZUSCOVITCH E. (1992), "Apprentissage technologique, réseaux économiques et appropriabilité des innovations ", Technologie et richesse des nations, Foray D. et Freeman C. (sous la direction de), Paris : Economica.

COLLINS H. (1980), Changing Order, London : Sage.

CORONINI R., JOLY P. B., DE LOOZE M.A. (1997), «Génomique : Une course à handicap ? ", Biofuturt, décembre, $n^{\circ} 173,14-17$.

DASGUPTA P. et DAVID P. (1994), "Toward a New Economics of Science", Research Policy, 23, 487-521.

EISENBERG R.S. (1996), "Intellectual Property at the Public-Private Divide : The Case of Large-scale DNA Sequencing ».
ELZEN B. (1988), Scientists and Rotors, PhD dissertation, University of Twente.

GALLOCHAT A. (1994), "Peut-on breveter le vivant?", La Recherche, $n^{\circ} 261$, janvier, 56-60.

- (1996), «Directive on Legal Protection of Biotechnological Inventions : Another or (last) Chance for Europe ». Patent World. 13-16.

GALLOU J.C. (1996), «Les enjeux d'une déclaration universelle sur la protection du génome humain ", Recueil Dalloz Sirey, $17^{\circ}$ cahier.

HERMITTE M.A. (1993), «Le projet génome humain : liberté de la recherche et démarche démocratique ", Revue Internationale du Droit Économique, 1, 29-51.

- (1993), «Lanimal à l'épreuve du droit des brevets ", Natures, Sciences, Socićtés, 1, 47-55.

JORDAN B. (1993), Voyage autour du génome. Le tour du monde en 80 labos, Les Éditions INSERM, Paris.

LABRUSSE-RIOU C. (1988), «Servitudes, servitudes », L'homme, la nature et le droit, Paris : Christian Bourgeois.

LATOUR B. et WOOLGAR S. (1979), Laboratory Life. Sage.

OCDE (1995), Le grand programme sur le génome humain, Paris.

PESTRE D. (1995), «Pour une histoire sociale et culturelle des sciences. Nouvelles définitions, nouveaux objets. nouvelles pratiques ", Annales ESC, 50 (3), 487-522.

RIFKIN J. (1997), The Bioted Century, New York : Norton Press. 